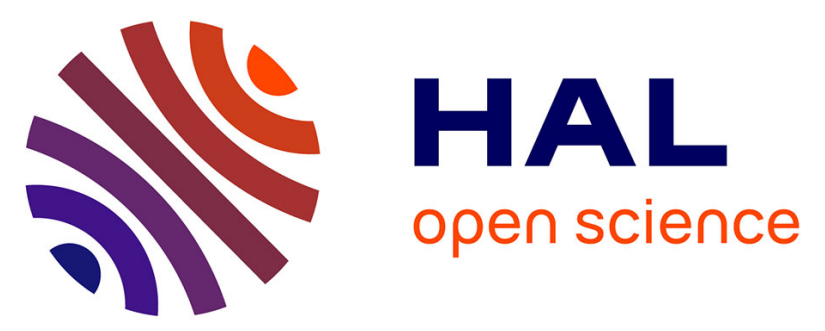

\title{
Scenario selection optimization in system engineering projects under uncertainty: a multi-objective ant colony method based on a learning mechanism
}

Majda Lachhab, Thierry Coudert, Cédrick Béler

\section{- To cite this version:}

Majda Lachhab, Thierry Coudert, Cédrick Béler. Scenario selection optimization in system engineering projects under uncertainty: a multi-objective ant colony method based on a learning mechanism. IEEM 2016, Dec 2016, Bali, Indonesia. pp. 1235-1239, 10.1109/IEEM.2016.7798075 hal-01635628

\section{HAL Id: hal-01635628 \\ https://hal.science/hal-01635628}

Submitted on 15 Nov 2017

HAL is a multi-disciplinary open access archive for the deposit and dissemination of scientific research documents, whether they are published or not. The documents may come from teaching and research institutions in France or abroad, or from public or private research centers.
L'archive ouverte pluridisciplinaire HAL, est destinée au dépôt et à la diffusion de documents scientifiques de niveau recherche, publiés ou non, émanant des établissements d'enseignement et de recherche français ou étrangers, des laboratoires publics ou privés. 


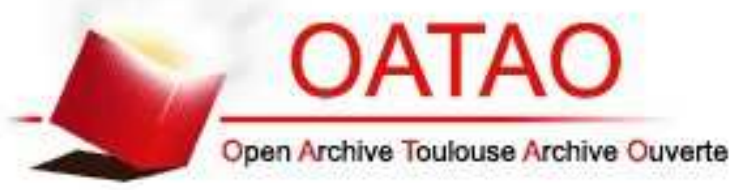

\section{Open Archive Toulouse Archive Ouverte (OATAO)}

OATAO is an open access repository that collects the work of Toulouse researchers and makes it freely available over the web where possible.

This is an author-deposited version published in: http://oatao.univ-toulouse.fr/ Eprints ID: 17845

\section{To cite this version:}

Lachhab, Majda and Coudert, Thierry and Béler, Cédrick Scenario selection optimization in system engineering projects under uncertainty: a multi-objective ant colony method based on a learning mechanism. (2016) In : IEEM 2016, 4 December 2016 - 7 December 2016 (Bali, Indonesia). 


\title{
Scenario Selection Optimization in System Engineering Projects Under Uncertainty: A Multi-Objective Ant Colony Method Based on a Learning Mechanism
}

\author{
M. Lachhab ${ }^{1}$, T. Coudert ${ }^{1}$, C. Béler ${ }^{1}$ \\ ${ }^{1}$ INP-ENIT, University of Toulouse, Tarbes, France \\ (majda.lachhab@enit.fr, thierry.coudert@enit.fr, cedrik.beler@enit.fr)
}

\begin{abstract}
This paper presents a multi-objective Ant Colony Optimization (MOACO) algorithm based on a learning mechanism (named MOACO-L) for the optimization of project scenario selection under uncertainty in a system engineering (SE) process. The objectives to minimize are the total cost of the project, its total duration and the global risk. Risk is considered as an uncertainty about task costs and task durations in the project graph. The learning mechanism aims to improve the MOACO algorithm for the selection of optimal project scenarios in a $\mathrm{SE}$ project by considering the uncertainties on the project objectives. The MOACO-L algorithm is then developed by taking into account ants' past experiences. The learning mechanism allows a better exploration of the search space and an improvement of the MOACO algorithm performance. To validate our approach, some experimental results are presented.
\end{abstract}

Keywords - Ant Colony Optimization, learning, multiobjective optimization, Pareto-optimality, risk, uncertainty, system engineering project.

\section{INTRODUCTION}

In an increased competitiveness environment, many companies are investing in design projects of new systems to meet customer requirements and the various strategic challenges of the market. The system engineering activities are: definition and design (system development), production, utilization and finally dismantling, recycling and renewing. In project management, the Project Time Management process completes the system engineering process by determining resources that fulfill the project objectives in terms of time, cost, risk, quality, performance, etc. That can be reached through cooperation between designers and project managers to obtain an integrated design process and project planning process by defining some coupling points between both processes [1] [2]. Previous works have proposed a hybrid multi-objective optimization approach based on Bayesian networks and evolutionary algorithms for an integrated product design and project design process [3]. The work presented in this paper matches this context. The problem we are dealing with is the selection of project scenarios in a resulting oriented graph using Pareto-front solutions (project scenarios). The graph includes the different design and project planning choices of a new system to deliver. The Fig. 1 summarizes the considered problem.

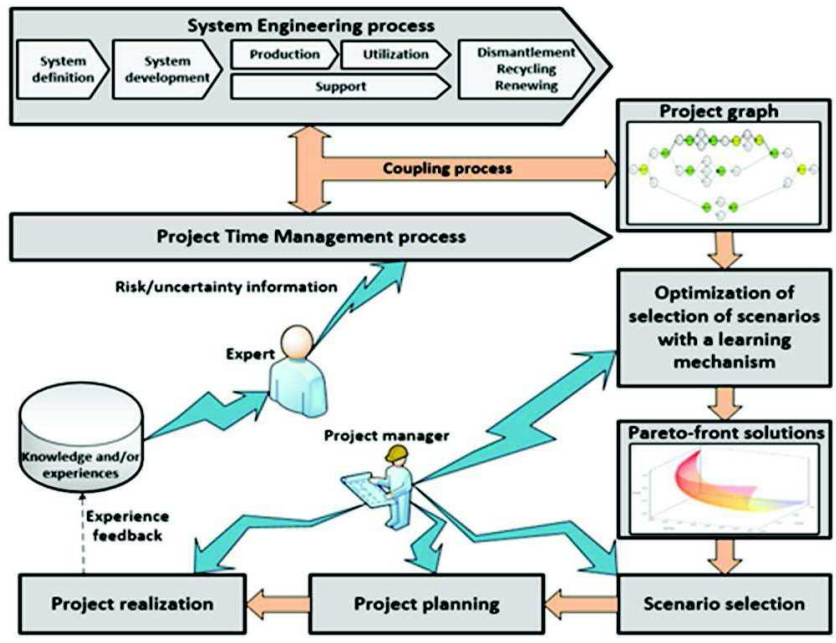

Fig. 1: Graphic representation for the developed problem.

Our first contribution consists in the development of a multi-objective ant colony optimization (MOACO) algorithm to select optimal scenarios taking into account their cost and delay, but also their associated risk assessment (risks are considered as uncertainties on costs and delays). The problem is then to optimize the selection of scenarios under uncertainty. The global uncertainty is a third objective to optimize (alongside cost and delay). Indeed, the selected scenario must be optimal regarding the risk associated with the increased duration and cost values of this scenario. The algorithm is based on artificial ants [4] which go through a task graph and make random choices to find a path. These choices are biased by the global attractivity of the paths already taken by ants in the previous iterations (experience feedback) and the local attractivity of the next task to reach. We adopt the MOACO metaheuristic [5] that allows to select high quality project scenarios of such relevant combinatorial optimization problem in a reasonable amount of time. In some works, the multi-objective optimization problems are handled by aggregating and weighting the multiple objectives depending on their relative importance [6]. However, the goal in this article is to offer to decision makers a set of non-dominated Pareto-optimal solutions to select a project to plan for the realization of the designed system. These solutions are optimal in the Pareto sense [7] [8]. In [9], the authors propose an ant colony optimization (ACO) algorithm in the case of the biobjective scheduling problem. There are many successful academic and industrial applications of ACO algorithms [10]. Some works have proved the power of ACO 
algorithms for solving both deterministic and probabilistic CPM/PERT networks by producing good optimal and suboptimal solutions [11]. Another work demonstrates the effectiveness of an ACO algorithm based on Ant Colony System (ACS) approach and Monte Carlo (MC) simulation for the maximization under uncertainty of the expected net present value (NPV) of cash flows in the case of scheduling multi-mode projects [12]. In the case of handling many objectives in real-world portfolio problems, the authors in [13] have developed a hybrid metaheuristic approach based on ACO and preference incorporation that provides high-quality portfolios in comparison with the leading metaheuristics that are dealing with Pareto-front solutions. Recently, the work presented in [14] has shown that a MOACO algorithm permits to manage the risks inherent to project tasks realization. Many risk management methods and tools are based on the probability and impact concepts to assess the risk using qualitative and quantitative approaches [15]. In [16], the authors argue the necessity to improve the management of uncertainty in projects by transforming the existing project risk management processes into project uncertainty management. Indeed, risk exists whenever uncertainty exists [17]. In [18] [19] [20], the risk is regarded as uncertainty about the task durations. It is taken into account by stochastic models. In [21], the uncertainty is represented by fuzzy logic based models. Recently, in [22] the authors have proposed a model for coupling project management, risk management and a lesson learning system (LLS) methodologies. However, they have not developed a formal model with the corresponding algorithms to enhance experience reuse in projects risk management. As noticed in this brief panorama, there are no studies addressing the multiobjective optimization of project scenarios selection integrating the risks as an objective, particularly in the case of system engineering projects.

Thus, our second contribution consists in developing an approach which allows to improve the MOACO algorithm performance by a learning mechanism (MOACO-L). In the standard ACO algorithms, every time an ant has to make a choice, it evaluates a probability to reach the next nodes. This probability depends on local attractivity of these next nodes and on global attractivity (i.e. pheromone trails corresponding to experience feedback) but not on previous choices. Therefore, we propose to couple the MOACO algorithm with a learning mechanism (MOACO-L). Thus, the ants can learn useful information from past runs in order to influence the future choices taking into account the previous ones.

The problem formalization and its associated project graph are given in section II. The proposed method, based on MOACO-L algorithm, is presented in section III. The algorithm is developed using Ruby language and the resulting tests of the improved MOACO algorithm within a learning process are discussed in section IV. Finally, conclusions and perspectives are described in section $\mathrm{V}$.

\section{PROBLEM FORMALIZATION}

The problem addressed in this paper is formulated by means of an acyclic and oriented project graph $G$. The graph $G$ is defined by $G=(N, A) . N$ is the set of nodes and $A$ is the set of arcs connecting these nodes and representing the precedence constraints between them. An example of project graph is represented in Fig. 2.

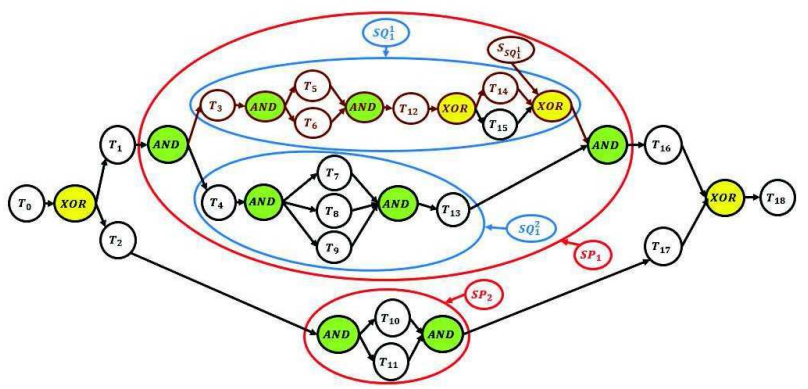

Fig. 2: Example of project graph.

The nodes of the graph represent tasks, conjunction logical operators $(A N D)$ and exclusive disjunction ones $(X O R)$. The first and last nodes of the graph are fictive tasks. Each task node is associated with a triplet $(C, D, R)$ corresponding to task cost value, its duration and the risk associated with these criterions. In our work, the risk is considered as an uncertainty about the achievement of project tasks. This uncertainty is related to the occurrence of undesirable events whose impacts increase the total cost of the project and/or its total duration. The uncertainties about the values of cost and duration criterions are represented as intervals. We assume that each interval has a minimum value being the nominal value of the criterion and a maximum value obtained from expert knowledge and/or past experiences [23]. A project $P$ is defined as a set of tasks and/or subprojects. $P$ is represented by:

$$
P=T^{P} U S P^{P}
$$

where, $T^{P}$ is the set of tasks of the project $P$ and $S P^{P}$ is the set of sub-projects of $P$. A sub-project is a sub-graph of the project graph $G$ that starts with a divergence node $A N D$ and ends with a convergence node $A N D$. A subproject is characterized by its cost, duration and the uncertainty about their values. A sub-project consists of parallel sub-sequences. A sub-sequence may be composed of other tasks, and/or other sequential sub-projects, and/or convergence and divergence $X O R$ nodes. A scenario $S$ represents a project graph path. $S$ is an ordered sequence of tasks $\left(T_{i}\right)$ and/or sub-projects $\left(S P_{j}\right)$ to realize. It is represented by:

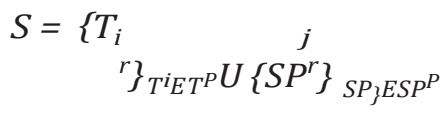

Each task $T_{i}$ and each sub-project $S P_{j}$ has a rank $r$ corresponding to their orders in a given scenario $S$ of the 
project $P$. The total cost $C_{S}$ of a scenario $S$ of a project $P$, is defined by:

$$
C_{S}=I_{T_{i} E S}\left[C_{T}, C_{T_{i}}^{+}\right]+I_{S P_{\}} E S}\left[C_{S P_{\}}}, C_{S P_{\}}}^{+}\right]=\left[C_{S}, C_{S}^{+}\right]
$$

The total duration $D_{S}$ of a scenario $S$ of a project $P$, is defined by:

$$
D_{S}=I_{T_{i} E S}\left[D_{T_{i}} D_{T_{i}}^{+}\right]+I_{S P_{\}} E S}\left[D_{S P_{\}}}, \stackrel{+}{D_{S P_{\gamma}}^{+}}\right]=\left[D_{S}, \stackrel{+}{D_{S}^{+}}\right]
$$

where $C_{S}^{-}$and $C_{S}^{+}$are respectively associated with the nominal cost of a scenario $S$ and its maximum cost in the case of occurrence of undesirable events. In the same way, $D_{S}^{-}$and $D_{S}^{+}$are respectively the nominal and maximal durations of the scenario $S .\left(C_{T_{i}}, C_{T_{i}}^{+}, D_{T_{i}}, \stackrel{+}{D_{T_{i}}^{+}}\right)$ and $\left(C_{S_{3}}, C_{S_{3}}, D_{S P_{\}}}, D_{S P_{\}}}\right)$correspond respectively to

tasks and sub-projects lower and upper bounds for cost and delay. Let $S P_{j}$ be a sub-project of $q$ parallel subsequences $S Q_{j}(k E\{1,-, q\})$. Let $S_{S Q^{k}}$ be, a scenario corresponding to the sub-sequence $k$. The duration of the scenario $S_{S Q^{k}}$ is given by:

$$
\begin{aligned}
D_{S Q_{\}}^{k}} & =I_{T_{h} E S_{S Q_{\}}}}\left[D_{T_{h}}, D_{T_{h}}^{+}\right]+I_{S P_{l} E S_{S Q_{\}}^{k}}}\left[D_{S P_{Y}} D_{S P_{Y}}^{+}\right] \\
& =D_{S Q_{\}}{ }^{k}}, D_{S_{S Q_{\}}^{k}}}^{+}
\end{aligned}
$$

Then, the duration of a sub-project $S P_{j}$ is given by considering that every task or sub-project will be planned at their respective earliest starting date:

$$
\begin{aligned}
& \left.\begin{array}{rllll}
D_{S P_{\}}}=M A X_{S Q_{\}}} & \left(\begin{array}{llll}
D_{S} &
\end{array}\right), M A X & k & \left(D_{S}{ }^{+}\right.
\end{array}\right) \\
& =\left[D_{S P_{\gamma}}, D_{S P_{\gamma}}\right]
\end{aligned}
$$

The Generalized Ordered Weighted Averaging operator (GOWA) [24] is a class of parametrized operators used to calculate the global risk $R_{S}$ of a scenario $S$ from uncertainties:

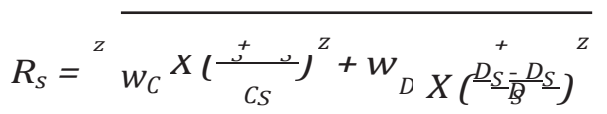

The expressions $\left(\frac{C_{S}{ }^{+} C}{S}\right)$ and $\left({ }_{C_{S}}^{\frac{D_{S}-D_{S}}{D_{S}}}\right)$ represent respectively the percentages related to the increase of the nominal values of cost and duration of realization of the scenario $S . w_{C}$ and $w_{D}$ are weights satisfying $w_{C}+w_{D}=$ 1 , and $z$ is a parameter such that $z E(-o o,+o o)$. In our model, $z=2$ (quadratic mean). Therefore, $R_{S}$ is the quadratic mean of the relative uncertainties on cost and delay.

\section{PROPOSED MOACO-L ALGORITHM}

The proposed MOACO-L algorithm is based on a single ant colony that will build solutions in the project graph by minimizing simultaneously the values of total cost, total duration and global risk. In each iteration, each ant builds its solution independently. Each arc $(i, j)$ of the graph $G$ contains three pheromone trails for each criterion of the triplet $(C, D, R)$. The quantity of pheromone on $(i, j)$ for each criterion $O E\{C, D, R\}$ is denoted by $r_{i j}^{O}$. All the ants of the colony are initialized from the first node of the project graph. Each ant $f$ makes a choice from a node $i$ to reach a node $j E N_{i}\left(N_{i}\right.$ 1s the set of neighbors of $i$ ) using the following probability formula:

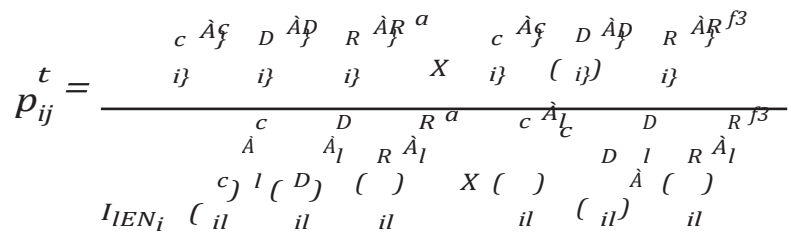

For each node $j E N_{i}$, we have:

$\underset{r J_{i j}}{C}=\begin{aligned} & q_{i_{C}} \\ & C_{T_{\}}}\end{aligned}$(9) $\quad r J_{i j}=\underbrace{q_{i_{D}}}_{D_{T_{j}}}$ (10) $\quad r J_{i j}=\frac{q_{i_{R}}}{R_{T_{3}}}$

where $r J_{i j}^{C}, r J_{i j}^{D}$ and $r J_{i j}^{R}$ represent respectively the local attractivity of a node $j$ regarding a node $i$, in terms of cost, duration and risk. ${ }_{D}<p^{C},<p_{R}^{D}$ and $<p^{R}$ are constants that ensure that $r J_{i j}, r J_{i j}$ and $r J_{i j}$ are upper or equal to one. Theses constants are calculated as follows:

$$
\begin{aligned}
& \begin{array}{c}
<p_{D}^{C}=M A X_{T_{T}} E_{T}^{P} \\
<M A T^{P}
\end{array}\left(C_{T}^{T}\right) \\
& <p^{R}=M A X_{T_{3}}{ }^{3} T^{P}\left(R_{T_{3}}{ }^{3}\right)
\end{aligned}
$$

The risk measurement for a task $T_{j}$ is denoted by $R_{T_{\}}}$and calculated using the GOWA operator:

$$
\begin{aligned}
& { }_{z}^{+} C_{T} \quad \stackrel{+}{D_{T}^{+}-D_{T}} \\
& R_{T_{\}}}=w_{C} X \frac{\left.C_{T_{3}}\right\}}{C_{T_{3}}}+w_{D} X \frac{\} \quad\}}{D_{T_{\}}}}
\end{aligned}
$$

$a$ and $f 3$ are the weights associated with the global attractivity of pheromone trails and to the local attractivity. At the end of each iteration, all the ants of the colony which have reached the last node of the $e_{D}$ graph dropeiaff three quantities itefionherem, $(i, j)$ belonging to their path (i.e. their scenario). The quantities of pheromone are initialized as $\left(\begin{array}{c}C \\ r_{O}, r_{O}, r_{O}\end{array}\right)$ and are updated at each iteration:

$$
\begin{gathered}
{ }^{C} r_{i j}(i t+1)=(1-p) X r_{i j}(i t)+I_{S E\left\{S_{i t t}\right\}} \\
r^{D}(i t+1)=(1-p) X r^{D}(i t)+I \quad \underline{\underline{1}}
\end{gathered}
$$




$$
r_{i j}^{R}(i t+1)=(1-p) X r_{i j}^{R}(i t)+\boldsymbol{I} \underset{S E\left\{S_{i t}\right\}}{1} \overline{R_{S}}
$$

$p$ is the evaporation rate of pheromone trails and $\left\{S_{i t}\right\}$ represents the set of scenarios made by all the ants of the colony at iteration (it). The weights $\grave{A}_{j}, \grave{A}_{j}$ and $\grave{A}_{j}$ are dynamic and vary at each new reached node. They allow to the MOACO-L algorithm to learn. The weights are defined by:

$$
\begin{aligned}
\dot{A}_{j}^{C} & =\frac{C p_{\}}^{C}}{C p_{\}}^{C}+C p_{\}}^{D}+C p_{\}}^{R}} \\
\dot{A}_{j}^{D} & =\frac{C p_{\}}^{D}}{C p_{\}}^{C}+C p_{\}}^{D}+C p_{\}}^{R}} \\
\dot{A}_{j}^{R} & =\frac{C p_{\}}^{k}}{\underbrace{}_{j}+p_{j}+C p_{\}}+C p_{\}}}
\end{aligned}
$$

with $\check{A}_{j}^{C}+\dot{A}_{j}^{D}+\hat{A}_{j}^{R}=1$. $C p_{j}^{C}, C p_{j}^{D}$ and $C p_{j}^{R}$ represent the consumed capital percentage of cost, duration and risk to guide the ant $f$ to reach the node $j E N_{i}$ using the probability $_{C}$ formula $_{R}(8)$. The aim of having dynamic weights $\left(\grave{A}_{j}, \grave{A}_{j}, \grave{A}_{j}\right)$ is to guide the ants in their choices by learning from their past choices. These percentages are calculated as follows:

$$
\begin{aligned}
& C p_{\boldsymbol{j}}^{C}=\frac{C_{j}^{g}}{C p C_{0}} \\
& C p_{j}^{D}=\frac{D_{\}}^{g}}{C p D_{0}}
\end{aligned}
$$

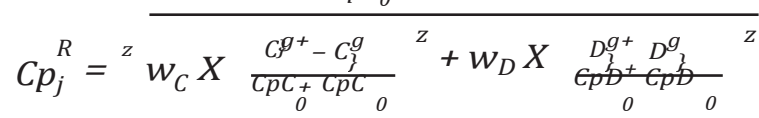

$$
\begin{aligned}
& C p C_{O}=\left[C p C_{O}, C p C_{O}^{+}\right] \\
& C p D_{O}=\left[C p D_{O}, C p D_{O}\right]
\end{aligned}
$$

$C p C_{O}$ and $C p D_{O}$ are respectively the initial cost and duration capitals and they are represented as intervals in order to model the capitals about uncertainties on cost and delay capitals. $C_{j}^{g}, D^{g}, G^{g+}$ and $D_{j}^{g+}$ are respectively the nominal and the maximal cumulated costs and durations at the node $j$, such as:

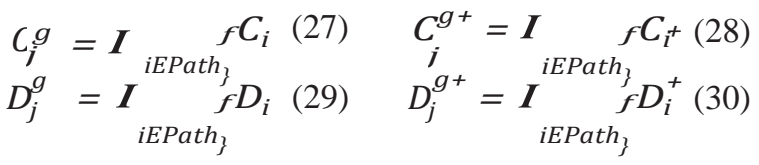

Path $_{j}^{t}$ is the path of the ant $f$ that includes all the visited nodes. Therefore, giving initial capitals to the ants permits to integrate a new bias into the probability formula (8). An ant which has consumed a great part of one of the three capitals will be influenced to choose next nodes that does not penalize this capital. Moreover, one can say that the ant learned from the path it uses in order to influence its future choices.

\section{EXPERIMENTS}

A set of experiments has been done to validate our method and attest the efficiency of experimefopesed

make a comparative analysis between MOACO and MOACO-L algorithms. The algorithms were developed in the Ruby language and executed on desktop computer (Intel ${ }^{\circledR}$ Core $^{\mathrm{TM}}$ i7 $3.6 \mathrm{GHz}$ processor). The set of experiments are made with a graph of 100 nodes organized following 23 sequential levels. Each level has a number of nodes that vary between 2 and 9 nodes. Every node of a level is connected to every node of the next level. The combinations give $44.10^{12}$ possible scenarios. The colony has 200 ants and the initial parameter settings

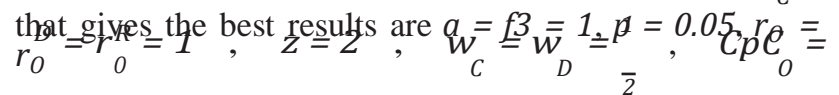
$[400000,417000], C p D_{O}=[740,790]$ and $\grave{A}_{O}^{C}=\grave{A}_{O}^{D}=$ $\grave{A}_{0}^{R}=\frac{1}{3}$. These parameters have been evaluated empirically. The Fig. 3 represents a comparison between MOACO and MOACO-L algorithms using mean performance (Mean Perf) and the standard deviation (StdDevPerf). MeanPerf and StdDevPerf are defined as follows:

\section{MeanPerf $=$ MeanC X MeanD XMeanR StdDevPerf $=S t d D e v C X$ StdDevDXStdDevR}

These values are calculated from the mean values of costs, durations and risks of the Pareto-optimal scenarios. The Pareto front is calculated at the end of each iteration (when the whole colony has reached the last node). The Fig. 3 represents the results of the 600 iterations for the MOACO and MOACO-L algorithms.

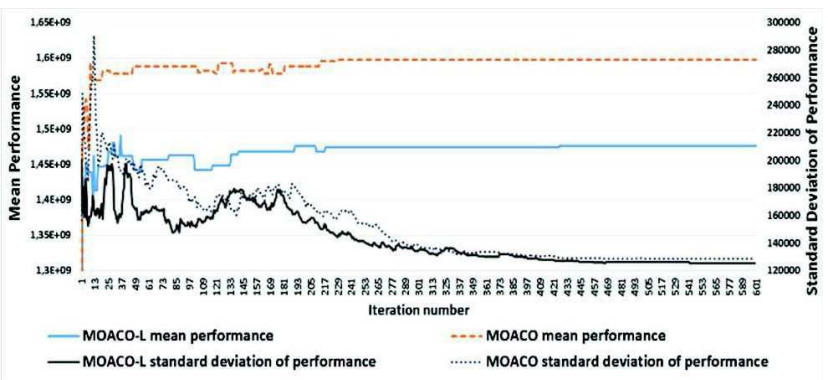

Fig. 3. Mean performance and standard deviation of performance of MOACO versus MOACO-L algorithms.

As shown in Fig. 3, the proposed MOACO-L algorithm gives better results than MOACO algorithm since it improves the mean performance in a reasonable amount of time. In fact, the MOACO-L algorithm generates Pareto-optimal solutions in 120.9 seconds versus 121.6 seconds for the MOACO algorithm. Furthermore, the learning mechanism allows the MOACO-L algorithm a better exploration of the search space because it stabilizes from the $426^{\text {th }}$ iteration rather than the MOACO algorithm which stabilizes earlier (from the $222^{\text {th }}$ iteration). Moreover, MOACO-L algorithm 
provides efficient solutions in terms of cost, duration and risk. In fact, the MOACO-L algorithm is more performant than the MOACO algorithm with almost a difference of $8.84 \%$ for mean performance and almost a difference of $2.73 \%$ for standard deviation of mean performance.

\section{CONCLUSION}

In this article, we have proposed a MOACO-L algorithm for the optimization of project scenario selection under uncertainty by integrating a learning mechanism. We have shown that MOACO-L algorithm gives better results than the standard one. In future works, we expect to develop a knowledge model that interfere with our proposed optimization model in order to learn in an intelligent manner from previous projects experiences the uncertainties on costs and delays. The knowledge about the events and their impact on delays and costs capitalized during previous projects or defined by an expert on risk management has to be learned by a simulation method and introduced into the MOACO-L algorithm in order to guide the optimization.

\section{REFERENCES}

[1] T. Coudert, E. Vareilles, L. Geneste, M. Aldanondo, and J. Abeille, "Proposal for an integrated Case Based Project Planning and system design process," Proceedings of the 2nd International Conference on Complex Systems Design \& Management, CSDM'2011, Springer Verlag, 7-9 September 2011, Paris (France).

[2] E. Vareilles, T. Coudert, M. Aldanondo, L. Geneste, and J. Abeille, "System design and project planning: Model and rules to manage their interactions," Integrated ComputerAided Engineering, vol. 22, no. 4, pp. 327-342, 2015.

[3] P. Pitiot, T. Coudert, L. Geneste, and C. Baron, "Hybridization of Bayesian networks and Evolutionary Algorithms for multi-objective optimization in an integrated product design and project management context," Engineering Applications of Artificial Intelligence, vol. 33 (5), pp. 830-843, 2010.

[4] M. Dorigo, "Optimization, Learning and Natural Algorithms" (in Italian), Ph.D. dissertation, Politecnico di Milano, Italy, 1992.

[5] M. Dorigo, and T. Stützle, Ant colony optimization. Cambridge, The MIT Press, 2004, pp. 25-64.

[6] M. Dorigo, M. Birattari, and T. Stützle, "Ant Colony Optimization, Artificial Ants as a computational Intelligence Technique," IEEE Computational Intelligence Magazine, Nov. 2006.

[7] O-L. De Weck, "Multiobjective Optimization: History and Promise," Third China-Japan-Korea Joint Symposium on Optimization of Structural and Mechanical Systems, 2004.

[8] D. Angus, and C. Woodward, "Multiple objective ant colony optimisation," Swarm Intelligence, vol. 3, no. 1, pp. 69-85, Mar. 2009.

[9] D. Merkle, and M. Middendorf, "Modeling the dynamics of ant colony optimization algorithms," Evolutionary Computation, vol. 10, no.3, pp.235-262, 2002.

[10] M. Dorigo, and T. Stützle, "Ant Colony Optimization: Overview and Recent Advances," In M. Gendreau and $Y$. Potvin (eds.), Handbook of Metaheuristics, 2nd edition,
International Series in Operations Research \& Management Science, Springer Verlag, New York, vol. 146, pp. 227-263, 2010.

[11] H. Abdallah, H-M. Emara, H-T. Dorrah, and A. Bahgat, "Using Ant Colony Optimization algorithm for solving project management problems," Expert Systems With Applications, vol. 36, no. 6, pp. 10004-10015, Aug. 2009.

[12] W-N. Chen, and J. Zhang, "Scheduling Multi-Mode Projects under Uncertainty to Optimize Cash Flows: A Monte Carlo Ant Colony System Approach," Journal of Computer Science and Technology, vol. 27, no. 5, pp. 950965, Sept. 2012.

[13] E. Fernandez, C. Gomez, G. Rivera, and L. Cruz-Reyes "Hybrid metaheuristic approach for handling many objectives and decisions on partial support in project portfolio optimisation," Information Sciences, vol. 315, pp. 102-122, Sept. 2015.

[14] P. Baroso, T. Coudert, E. Villeneuve, and L. Geneste, "Multi-objective optimization and risk assessment in system engineering project planning by Ant Colony Algorithm," 2014 IEEE International Conference on Industrial Engineering Management, pp. 438-442, KualaLumpur, Malaysia, Dec. 2014.

[15] C. Fang, and F. Marle, "A simulation-based risk network model for decision support in project risk management," Decision Support Systems, vol. 52, no. 3, pp. 635-644, Feb. 2012.

[16] S. Ward, and C. Chapman, "Transforming project risk management into project uncertainty management," International Journal of Project Management, vol. 21, no. 2, pp. 97-105, Feb. 2003.

[17] M. Better, and F. Glover, "Simulation Optimization: applications in Risk Management," International Journal of Information Technology \& Decision Making, vol. 7, no. 4, pp. 571-587, Dec. 2008.

[18] W. Sobel, A. Aleskovski, P. Hand, Z. Liu, M. Bishopp, A. Honikman, L. Anderson, and R. Close, "Method and apparatus for network-based portfolio management and risk-analysis," Patent US 7596523 B2, October 2004.

[19] E. Elisa Johanna Maria Creemers, Y-M. Pinto, and A. Johanna Marina Tijsen, "Means and methods for counteracting, preventing and/or determining heart failure, or a risk of heart failure," Patent EP 2907879 A3, May 2012.

[20] J-J. Bourne, C. Annibale, R. Behara, C. George Kenneth Copland, D-P. Ferrick, T. Farrell, and N. Scott, "Method and system to determine auto insurance risk," Patent WO 2014059208 A3, April 2014.

[21] I. Kaya, and C. Karhaman, "Development of fuzzy process accuracy index for decision making problems," Special Issue on Modelling Uncertainty, vol. 180, no. 6, March 2010, pp. 861-872.

[22] V. Manotas-Niño, P. Clermont, L. Geneste, and A-X. Halabi, "Towards a Model of Integration between Risk Management and Lesson Learning System for Project Management," presented at the 6th International Conference on Industrial Engineering and Systems Management, Seville, Spain, 2015.

[23] E. Villeneuve, C. Béler, F. Peres, L. Geneste, and E. Reubrez, "Decision-Support Methodology to Assess Risk in End-of-Life Management of Complex Systems," IEEE Systems Journal, no. 99, pp. 1-10, ISSN 1932-8184, 2016.

[24] R. Yager, "Generalized OWA aggregation operators," Fuzzy Optimization and Decision Making, Vol. 3 (1), pp. 93-107. 2004. 